\author{
T.Sh. Kal'menov, G.D. Arepova \\ Institute of Mathematics and Mathematical Modeling, Almaty, Kazakhstan \\ (E-mail: kalmenov@math.kz)
}

\title{
A criterion for the existence of soliton solutions of telegraph equation
}

In this paper we consider a telegraph equation. In the case of a rectangular domain for the Cauchy potential the lateral boundary conditions obtained. When considering the equation in the first quadrant a criterion for the existence of soliton solutions is obtained.

Keywords: telegraph equation, telegraph potential, fundamental solution, soliton solution, nonlocal boundary conditions, convolution.

\section{Introduction}

Many studies have been devoted to the study of classical potentials: the Newton potential, the volume heat potential and the wave potential. In the equations of mathematical physics Newton's potentials are used to solve classical problems (the Dirichlet problem, Neumann problem, Robin problem) for the Laplace equation and other elliptic equations. It should be noted that for the first time the exact nonlocal boundary conditions of the Newton potential, the volume heat potential and the wave potential have been found recently [1-3]. After, boundary conditions of surface potentials that satisfy homogeneous equations were studied [4,5]. Further applying these results, the boundary conditions for the volume elliptic-parabolic potential were found and so on $[6-19]$.

\section{Boundary conditions of Telegraph Equation}

In the band $\Omega=\left\{t \geq 0,0 \leq x \leq \frac{1}{2}\right\}$ we select a limited subdomain $\Omega_{1}=\left\{0 \leq t \leq \frac{1}{2}, 0 \leq x \leq \frac{1}{2}\right\}$ and consider the Cauchy problem for a one-dimensional telegraph equation

$$
\begin{gathered}
L u(x, t)=\frac{\partial^{2} u(x, t)}{\partial t^{2}}-\frac{\partial^{2} u(x, t)}{\partial x^{2}}-\lambda u(x, t)=f(x, t) ; \\
\left.u(x, t)\right|_{t=0}=0 \\
\left.\frac{\partial u(x, t)}{\partial t}\right|_{t=0}=0 .
\end{gathered}
$$

In the characteristic coordinates $\xi=x+t, \eta=x-t$ the band $\Omega$ turns into a band $\widetilde{\Omega}$, and the subdomain $\Omega_{1}$ turns into a subdomain $\widetilde{\Omega}_{1}$ with bounded segments:

$$
\begin{gathered}
A_{0} B_{0}: \eta=\xi, \quad 0 \leq \xi \leq \frac{1}{2} ; \quad A_{0} A: \eta=-\xi, \quad 0 \leq \xi \leq \frac{1}{2} \\
B_{0} B: \eta=1-\xi, \quad \frac{1}{2} \leq \xi \leq 1 ; \quad A B: \eta=\xi-1, \quad \frac{1}{2} \leq \xi \leq 1,
\end{gathered}
$$

as $t \geq 0$, then $\eta \leq \xi$. Equation (1) also turns into equation

$$
L u(\xi, \eta)=\frac{\partial^{2} u(\xi, \eta)}{\partial \xi \partial \eta}+\frac{\lambda}{4} u(\xi, \eta)=f_{1}(\xi, \eta)
$$

and the Cauchy data (2) and (3) are expressed in

$$
\begin{gathered}
\left.u(\xi, \eta)\right|_{\eta=\xi}=0 \\
\left.\left(\frac{\partial u(\xi, \eta)}{\partial \xi}-\frac{\partial u(\xi, \eta)}{\partial \eta}\right)\right|_{\eta=\xi}=0
\end{gathered}
$$


It is well known that the Riemann function $R\left(\xi, \eta, \xi_{1}, \eta_{1}\right)$ (see [20;92]) of the telegraph equation (4) is representable in the form

$$
R\left(\xi, \eta, \xi_{1}, \eta_{1}\right)=J_{0}\left(\sqrt{\lambda\left(\xi-\xi_{1}\right)\left(\eta-\eta_{1}\right)}\right),
$$

where $J_{0}(z)$ is a zero-order Bessel function (see [20; 91]).

A fundamental solution of the Cauchy problem (4)-(6) in the domain $\widetilde{\Omega}$ is given by the formula:

$$
\varepsilon\left(\xi-\xi_{1}, \eta_{1}-\eta\right)=-\theta\left(\xi-\xi_{1}\right) \theta\left(\eta_{1}-\eta\right) \times R\left(\xi, \eta, \xi_{1}, \eta_{1}\right) .
$$

In contrast to $[21 ; 256]$, here the fundamental solution is taken with a negative sign and the second argument $\left(\eta_{1}-\eta\right)$ in accordance with the domain $\widetilde{\Omega}$.

The telegraph potential in the domain $\widetilde{\Omega}$ is called the integral

$$
\begin{gathered}
u(\xi, \eta)=\varepsilon * f=\int_{\widetilde{\Omega}} \varepsilon\left(\xi-\xi_{1}, \eta_{1}-\eta\right) f\left(\xi_{1}, \eta_{1}\right) d \xi_{1} d \eta_{1}= \\
=\int_{\eta}^{\xi} d \xi_{1} \int_{\xi_{1}}^{\eta} R\left(\xi, \eta, \xi_{1}, \eta_{1}\right) f\left(\xi_{1}, \eta_{1}\right) d \eta_{1}= \\
=\int_{\eta}^{\xi} d \xi_{1} \int_{\xi_{1}}^{\eta} J_{0}\left(\sqrt{\lambda\left(\xi-\xi_{1}\right)\left(\eta-\eta_{1}\right)}\right) f\left(\xi_{1}, \eta_{1}\right) d \eta_{1} .
\end{gathered}
$$

It is easy to verify that the telegraph potential (9) satisfies the homogeneous initial Cauchy conditions for $\eta=\xi, 0 \leq \xi \leq 1 / 2$, i.e.

$$
\begin{gathered}
\left.u(\xi, \eta)\right|_{\eta=\xi}=0 \\
\left.\left(\frac{\partial u(\xi, \eta)}{\partial \xi}-\frac{\partial u(\xi, \eta)}{\partial \eta}\right)\right|_{\eta=\xi}=0
\end{gathered}
$$

and equation (4) (see [21]).

Let us find a lateral boundary conditions of the telegraph potential (9) for $A_{0} A: \xi=-\eta, \quad 0 \leq \xi \leq \frac{1}{2}$ and $B_{0} B: \xi=1-\eta, \quad \frac{1}{2} \leq \xi \leq 1$, which is equivalent to $x=0$, and $x=\frac{1}{2}$ in the original coordinates $(x, t)$.

Theorem 1. Let $f(\xi, \eta) \in C^{1}(\widetilde{\widetilde{\Omega}})$, then the telegraph potential $u(\xi, \eta) \in C^{2}(\widetilde{\widetilde{\Omega}})$ satisfies the following lateral boundary conditions:

$$
\begin{gathered}
\left.N[u]\right|_{A_{0} A}=\left.N[u]\right|_{\xi=-\eta}=\int_{0}^{\xi} J_{0}\left(\sqrt{4 \lambda\left(\xi-\xi_{1}\right)^{2}}\right) \frac{\partial u}{\partial \eta_{1}}\left(\xi_{1},-\eta_{1}\right) d \xi_{1}+ \\
+2 \lambda \int_{0}^{\xi} \frac{J_{1}\left(\sqrt{-4 \lambda\left(\xi-\xi_{1}\right)^{2}}\right)}{\left(\sqrt{-4 \lambda\left(\xi-\xi_{1}\right)^{2}}\right)} u\left(\xi_{1},-\xi_{1}\right) d \xi_{1}=0 ; \\
\left.N[u]\right|_{B_{0} B}=\left.N[u]\right|_{\xi=1-\eta}=-\int_{\frac{1}{2}}^{\xi} J_{0}\left(\sqrt{-4 \lambda\left(\xi-\xi_{1}\right)^{2}}\right) \frac{\partial u}{\partial \xi_{1}}\left(\xi_{1}, 1-\xi_{1}\right) d \xi_{1}+ \\
+2 \lambda \int_{\frac{1}{2}}^{\xi} \frac{J_{1}\left(\left(\sqrt{-4 \lambda\left(\xi-\xi_{1}\right)^{2}}\right)\right)}{\left(\sqrt{-4 \lambda\left(\xi-\xi_{1}\right)^{2}}\right)}\left(\xi-\xi_{1}\right) u\left(\xi_{1}, 1-\xi_{1}\right) d \xi_{1}=0,
\end{gathered}
$$

where $J_{1}(z)$ is a Bessel function of the first order.

Conversely, if $u(\xi, \eta) \in C^{2}(\widetilde{\Omega})$ is a solution of the telegraph equation (4), satisfying the initial conditions (5)-(6) and the lateral boundary conditions (12)-(13), then $u(\xi, \eta)$ is given by the telegraph potential (9).

We note that for $\lambda=0$ the lateral boundary conditions of the telegraph potential coincide with the boundary conditions of the one-dimensional wave potential which is given in [3].

Proof. We continue the function $f(\xi, \eta)$ outside of the square $\widetilde{\Omega}_{1}$ with zero, i.e. $f(\xi, \eta) \equiv 0$ in $R^{2} / \widetilde{\Omega}_{1}$. Then the telegraph potential

$$
u(\xi, \eta)=\varepsilon * f=\int_{\eta}^{\xi} d \xi_{1} \int_{\xi_{1}}^{\eta} R\left(\xi, \eta, \xi_{1}, \eta_{1}\right) f\left(\xi_{1}, \eta_{1}\right) d \eta_{1}
$$


gives a solution of equation (4) for all $(\xi, \eta) \in R^{2}$ and $u(\xi, \eta) \in C^{2}(\overline{\widetilde{\Omega}})$ satisfies the homogeneous initial Cauchy conditions (5)-(6) for the whole straight line $\xi=\eta,-\infty<\xi<+\infty$. The value of the function $u(\xi, \eta)$ at the point $(\xi, \eta)$ is determined by the value of $f\left(\xi_{1}, \eta_{1}\right)$ in the characteristic triangle, i.e. $\triangle_{\xi, \eta}=\left\{\eta \leq \xi_{1} \leq \xi, \eta \leq \eta_{1} \leq \xi_{1}\right\}$.

Therefore the value of the function $u(\xi, \eta)$ on $\left.u\right|_{A_{0} A=\eta=-\xi}=u(\xi,-\xi)$ is defined by the formula

$$
u(\xi,-\xi)=\int_{-\xi}^{\xi} d \xi_{1} \int_{\xi_{1}}^{-\xi} R\left(\xi,-\xi, \xi_{1}, \eta_{1}\right) f\left(\xi_{1}, \eta_{1}\right) d \eta_{1} .
$$

As $f \equiv 0$ outside $\xi_{1} \leq 0$ and $\eta_{1} \leq-\xi_{1}$, then the integral (14) takes the form

$$
\begin{gathered}
\left.u\right|_{A_{0} A}=u(\xi,-\xi)=\int_{0}^{\xi} d \xi_{1} \int_{\xi_{1}}^{-\xi_{1}} R\left(\xi,-\xi, \xi_{1}, \eta_{1}\right) f\left(\xi_{1}, \eta_{1}\right) d \eta_{1}= \\
=\int_{0}^{\xi} d \xi_{1} \int_{\xi_{1}}^{-\xi_{1}} J_{0}\left(\sqrt{\lambda\left(\xi-\xi_{1}\right)\left(-\xi-\eta_{1}\right)}\right) f\left(\xi_{1}, \eta_{1}\right) d \eta_{1} .
\end{gathered}
$$

Now, in (15) instead of the function $f\left(\xi_{1}, \eta_{1}\right)$ we put $\frac{\partial^{2} u\left(\xi_{1}, \eta_{1}\right)}{\partial \xi_{1} \partial \eta_{1}}+\frac{\lambda}{4} u\left(\xi_{1}, \eta_{1}\right)$, i.e.

$$
\begin{gathered}
u(\xi,-\xi)=\int_{0}^{\xi} d \xi_{1} \int_{\xi_{1}}^{-\xi_{1}} J_{0}\left(\sqrt{\lambda\left(\xi-\xi_{1}\right)\left(-\xi-\eta_{1}\right)}\right)\left(\frac{\partial^{2} u\left(\xi_{1}, \eta_{1}\right)}{\partial \xi_{1} \partial \eta_{1}}+\frac{\lambda}{4} u\left(\xi_{1}, \eta_{1}\right)\right) d \eta_{1}= \\
=I_{1}+I_{2},
\end{gathered}
$$

where

$$
\begin{aligned}
& I_{1}=\frac{\lambda}{4} \int_{0}^{\xi} d \xi_{1} \int_{\xi_{1}}^{-\xi_{1}} J_{0}\left(\sqrt{\lambda\left(\xi-\xi_{1}\right)\left(-\xi-\eta_{1}\right)}\right) u\left(\xi_{1}, \eta_{1}\right) d \eta_{1} \\
& I_{2}=\int_{0}^{\xi} d \xi_{1} \int_{\xi_{1}}^{-\xi_{1}} J_{0}\left(\sqrt{\lambda\left(\xi-\xi_{1}\right)\left(-\xi-\eta_{1}\right)}\right) \frac{\partial^{2} u\left(\xi_{1}, \eta_{1}\right)}{\partial \xi_{1} \partial \eta_{1}} d \eta_{1} .
\end{aligned}
$$

Integrating by parts the integral $I_{2}$, we obtain

$$
\begin{gathered}
I_{2}=\int_{0}^{\xi}\left(\left.J_{0}\left(\sqrt{\lambda\left(\xi-\xi_{1}\right)\left(-\xi-\eta_{1}\right)} \frac{\partial u\left(\xi_{1}, \eta_{1}\right)}{\partial \xi_{1}}\right)\right|_{\xi_{1}} ^{-\xi_{1}} d \xi_{1}-\right. \\
-\int_{0}^{\xi} d \xi_{1} \int_{\xi_{1}}^{-\xi_{1}} \frac{\partial}{\partial \eta_{1}} J_{0}\left(\sqrt{\lambda\left(\xi-\xi_{1}\right)\left(-\xi-\eta_{1}\right)}\right) \frac{\partial u\left(\xi_{1}, \eta_{1}\right)}{\partial \xi_{1}} d \eta_{1}= \\
=\int_{0}^{\xi} J_{0}\left(\sqrt{\lambda\left(\xi-\xi_{1}\right)\left(\xi_{1}-\xi\right)}\right) \frac{\partial u\left(\xi_{1},-\xi_{1}\right)}{\partial \xi_{1}} d \xi_{1}- \\
-\int_{0}^{\xi} J_{0}\left(\sqrt{\lambda\left(\xi-\xi_{1}\right)\left(-\xi-\xi_{1}\right)}\right) \frac{\partial u\left(\xi_{1}, \xi_{1}\right)}{\partial \xi_{1}} d \xi_{1}- \\
-\int_{0}^{\xi} d \xi_{1} \int_{\xi_{1}}^{-\xi_{1}} \frac{\partial}{\partial \eta_{1}} J_{0}\left(\sqrt{\lambda\left(\xi-\xi_{1}\right)\left(-\xi-\eta_{1}\right)}\right) \frac{\partial u\left(\xi_{1}, \eta_{1}\right)}{\partial \xi_{1}} d \eta_{1} .
\end{gathered}
$$

From the initial Cauchy condition (5) it follows that $\frac{\partial u\left(\xi_{1}, \xi_{1}\right)}{\partial \xi_{1}}=0$. Taking this into account, the integral $I_{2}$ can be rewritten as

$$
\begin{gathered}
I_{2}=I_{2,1}-I_{2,2}=\int_{0}^{\xi} J_{0}\left(\sqrt{\lambda\left(\xi-\xi_{1}\right)\left(\xi_{1}-\xi\right)}\right) \frac{\partial u\left(\xi_{1},-\xi_{1}\right)}{\partial \xi_{1}} d \xi_{1}- \\
-\int_{0}^{\xi} d \xi_{1} \int_{\xi_{1}}^{-\xi_{1}} \frac{\partial}{\partial \eta_{1}} J_{0}\left(\sqrt{\lambda\left(\xi-\xi_{1}\right)\left(-\xi-\eta_{1}\right)}\right) \frac{\partial u\left(\xi_{1}, \eta_{1}\right)}{\partial \xi_{1}} d \eta_{1} .
\end{gathered}
$$

In the integral $I_{2,2}$ we change the order of integration and the limits in the domain $\eta \geq 0: 0 \leq \eta_{1} \leq \xi$ and in the domain $\eta \leq 0:-\xi \leq \eta_{1} \leq 0$, then we obtain 


$$
\begin{aligned}
I_{2,2} & =\int_{0}^{\xi} d \xi_{1} \int_{\xi_{1}}^{-\xi_{1}} \frac{\partial}{\partial \eta_{1}} J_{0}\left(\sqrt{\lambda\left(\xi-\xi_{1}\right)\left(-\xi-\eta_{1}\right)}\right) \frac{\partial u\left(\xi_{1}, \eta_{1}\right)}{\partial \xi_{1}} d \eta_{1}= \\
= & \int_{-\xi}^{0} d \eta_{1} \int_{\xi}^{-\eta_{1}} \frac{\partial}{\partial \eta_{1}} J_{0}\left(\sqrt{\lambda\left(\xi-\xi_{1}\right)\left(-\xi-\eta_{1}\right)}\right) \frac{\partial u\left(\xi_{1}, \eta_{1}\right)}{\partial \xi_{1}} d \xi_{1}+ \\
& +\int_{0}^{\xi} d \eta_{1} \int_{\xi}^{\eta_{1}} \frac{\partial}{\partial \eta_{1}} J_{0}\left(\sqrt{\lambda\left(\xi-\xi_{1}\right)\left(-\xi-\eta_{1}\right)}\right) \frac{\partial u\left(\xi_{1}, \eta_{1}\right)}{\partial \xi_{1}} d \xi_{1} .
\end{aligned}
$$

Using the formula $\frac{\partial}{\partial z} J_{0}(z)=-J_{1}(z)$ (see [21]), we conclude that

$$
\begin{gathered}
\frac{\partial}{\partial \eta_{1}} J_{0}\left(\sqrt{\lambda\left(\xi-\xi_{1}\right)\left(-\xi-\eta_{1}\right)}\right)=\frac{\partial}{\partial z} J_{0}(z) \frac{\partial z}{\partial \eta_{1}}= \\
=-J_{1}(z) \frac{\partial}{\partial \eta_{1}}\left(\sqrt{\lambda\left(\xi-\xi_{1}\right)\left(-\xi-\eta_{1}\right)}\right)=J_{1}(z) \frac{\lambda\left(\xi-\xi_{1}\right)}{2 \sqrt{\lambda\left(\xi-\xi_{1}\right)\left(-\xi-\eta_{1}\right)}} .
\end{gathered}
$$

Taking into account the last relation from (21), integrating by parts the integral $I_{2,2}$, we find

$$
\begin{aligned}
& I_{2,2}=\left.\frac{\lambda}{2} \int_{-\xi}^{0} \frac{J_{1}\left(\sqrt{\lambda\left(\xi-\xi_{1}\right)\left(-\xi-\eta_{1}\right)}\right)\left(\xi-\xi_{1}\right)}{\sqrt{\lambda\left(\xi-\xi_{1}\right)\left(-\xi-\eta_{1}\right)}} u\left(\xi_{1}, \eta_{1}\right)\right|_{\xi} ^{-\eta_{1}} d \eta_{1}+ \\
& \quad+\left.\frac{\lambda}{2} \int_{0}^{\xi} \frac{J_{1}\left(\sqrt{\lambda\left(\xi-\xi_{1}\right)\left(-\xi-\eta_{1}\right)}\right)\left(\xi-\xi_{1}\right)}{\sqrt{\lambda\left(\xi-\xi_{1}\right)\left(-\xi-\eta_{1}\right)}} u\left(\xi_{1}, \eta_{1}\right)\right|_{\xi} ^{\eta_{1}} d \eta_{1}- \\
& -\int_{-\xi}^{0} d \eta_{1} \int_{\xi}^{-\eta_{1}} \frac{\partial^{2}}{\partial \xi_{1} \partial \eta_{1}} J_{0}\left(\sqrt{\left.\lambda\left(\xi-\xi_{1}\right)\left(-\xi-\eta_{1}\right)\right)} u\left(\xi_{1}, \eta_{1}\right) d \eta_{1}+\right. \\
& -\int_{0}^{\xi} d \eta_{1} \int_{\xi}^{\eta_{1}} \frac{\partial^{2}}{\partial \xi_{1} \partial \eta_{1}} J_{0}\left(\sqrt{\left.\lambda\left(\xi-\xi_{1}\right)\left(-\xi-\eta_{1}\right)\right)} u\left(\xi_{1}, \eta_{1}\right) d \eta_{1}=\right. \\
& \quad=\frac{\lambda}{2} \int_{-\xi}^{0} \frac{J_{1}\left(\sqrt{-\lambda\left(\xi+\eta_{1}\right)\left(\xi+\eta_{1}\right)}\right)\left(\xi+\eta_{1}\right)}{\sqrt{-\lambda\left(\xi+\eta_{1}\right)\left(\xi+\eta_{1}\right)}} u\left(-\eta_{1}, \eta_{1}\right) d \eta_{1}+ \\
& +\int_{-\xi}^{0} d \eta_{1} \int_{\xi}^{-\eta_{1}} \frac{\partial^{2}}{\partial \xi_{1} \partial \eta_{1}} J_{0}\left(\sqrt{\left.\lambda\left(\xi-\xi_{1}\right)\left(-\xi-\eta_{1}\right)\right)} u\left(\xi_{1}, \eta_{1}\right) d \eta_{1}-\right. \\
& \quad-\int_{0}^{\xi} d \eta_{1} \int_{\xi}^{\eta_{1}} \frac{\partial^{2}}{\partial \xi_{1} \partial \eta_{1}} J_{0}\left(\sqrt{\left.\lambda\left(\xi-\xi_{1}\right)\left(-\xi-\eta_{1}\right)\right)} u\left(\xi_{1}, \eta_{1}\right) d \eta_{1} .\right.
\end{aligned}
$$

In the first integral of (22) replacing the variables $-\eta_{1}=\xi_{1}, \eta_{1}=\xi$, we have

$$
I_{2,2}^{1}=\frac{\lambda}{2} \int_{0}^{\xi} \frac{J_{1}\left(\sqrt{-\lambda\left(\xi-\xi_{1}\right)^{2}}\right)}{\sqrt{-\lambda\left(\xi-\xi_{1}\right)^{2}}}\left(\xi-\xi_{1}\right) u\left(\xi_{1},-\xi_{1}\right) d \xi_{1} .
$$

Taking into account the integrals $I_{2,1}, I_{2,2}^{1}$ and formulas (17), (22), we have that

$$
\begin{gathered}
\left.u\right|_{A_{0} A}=u(\xi,-\xi)=\int_{0}^{\xi} J_{0} \sqrt{\lambda\left(\xi-\xi_{1}\right)\left(\xi_{1}-\xi\right)} \frac{\partial u\left(\xi_{1},-\xi_{1}\right)}{\partial \xi_{1}} d \xi_{1}- \\
-\frac{\lambda}{2} \int_{0}^{\xi} \frac{J_{1}\left(\sqrt{-\lambda\left(\xi-\xi_{1}\right)^{2}}\right)}{\sqrt{-\lambda\left(\xi-\xi_{1}\right)^{2}}}\left(\xi-\xi_{1}\right) u\left(\xi_{1},-\xi_{1}\right) d \xi_{1}+ \\
+\int_{0}^{\xi} d \xi_{1} \int_{\xi_{1}}^{-\xi_{1}}\left(\frac { \partial ^ { 2 } } { \partial \xi _ { 1 } \partial \eta _ { 1 } } J _ { 0 } \left(\sqrt{\left.\lambda\left(\xi-\xi_{1}\right)\left(\eta-\eta_{1}\right)\right)}+\frac{\lambda}{4} J_{0}\left(\sqrt{\left.\lambda\left(\xi-\xi_{1}\right)\left(\eta-\eta_{1}\right)\right)}\right) u\left(\xi_{1}, \eta_{1}\right) d \eta_{1} .\right.\right.
\end{gathered}
$$

As $J_{0}\left(\sqrt{\left.\lambda\left(\xi-\xi_{1}\right)\left(\eta-\eta_{1}\right)\right)}\right.$ is the Riemann function of the telegraph equation (4), then 


$$
\frac{\partial^{2}}{\partial \xi_{1} \partial \eta_{1}} J_{0}\left(\sqrt{\left.\lambda\left(\xi-\xi_{1}\right)\left(\eta-\eta_{1}\right)\right)}+\frac{\lambda}{4} J_{0}\left(\sqrt{\left.\lambda\left(\xi-\xi_{1}\right)\left(\eta-\eta_{1}\right)\right)}=0 .\right.\right.
$$

Therefore, we have

$$
\begin{aligned}
\left.u\right|_{A_{0} A} & =u(\xi,-\xi)=\int_{0}^{\xi} J_{0} \sqrt{\lambda\left(\xi-\xi_{1}\right)\left(\xi_{1}-\xi\right)} \frac{\partial u\left(\xi_{1},-\xi_{1}\right)}{\partial \xi_{1}} d \xi_{1}- \\
& -\frac{\lambda}{2} \int_{0}^{\xi} \frac{J_{1}\left(\sqrt{-\lambda\left(\xi-\xi_{1}\right)^{2}}\right)}{\sqrt{-\lambda\left(\xi-\xi_{1}\right)^{2}}}\left(\xi-\xi_{1}\right) u\left(\xi_{1},-\xi_{1}\right) d \xi_{1} .
\end{aligned}
$$

It is easy to verify that the total derivative

$$
\frac{d}{d \xi_{1}} u\left(\xi_{1},-\xi_{1}\right)=\left.\left(\frac{\partial u\left(\xi_{1},-\xi_{1}\right)}{\partial \xi_{1}}-\frac{\partial u\left(\xi_{1},-\xi_{1}\right)}{\partial \eta_{1}}\right)\right|_{\eta=-\xi_{1}},
$$

then

$$
\frac{\partial u\left(\xi_{1},-\xi_{1}\right)}{\partial \xi_{1}}=\frac{d u\left(\xi_{1},-\xi_{1}\right)}{d \xi}+\frac{\partial u\left(\xi_{1},-\xi_{1}\right)}{\partial \eta_{1}} .
$$

Taking this into account, from (25) integrating by parts, we obtain

$$
\begin{gathered}
\int_{0}^{\xi} J_{0}\left(\left.\sqrt{\left.\lambda\left(\xi-\xi_{1}\right)\left(\xi_{1}-\xi\right)\right)}\left(\frac{d u\left(\xi_{1},-\xi_{1}\right)}{d \xi}+\frac{\partial u\left(\xi_{1},-\xi_{1}\right)}{\partial \eta_{1}}\right)\right|_{\eta_{1}=-\xi_{1}} d \xi_{1}=\right. \\
=\int_{0}^{\xi} J_{0}\left(\left.\sqrt{\left.\lambda\left(\xi-\xi_{1}\right)^{2}\right)} \frac{\partial u\left(\xi_{1}, \eta_{1}\right)}{\partial \eta_{1}}\right|_{\eta_{1}=-\xi_{1}} d \xi_{1}+\right. \\
+J_{0}\left(\left.\sqrt{\left.\lambda\left(\xi-\xi_{1}\right)^{2}\right)} u\left(\xi_{1},-\xi_{1}\right)\right|_{\xi_{1}=\xi}-\int_{0}^{\xi} \frac{\partial}{\partial \xi_{1}} J_{0}\left(\sqrt{\left.\lambda\left(\xi-\xi_{1}\right)^{2}\right)} u\left(\xi_{1},-\xi_{1}\right) d \xi_{1}=\right.\right. \\
=\frac{1}{2} \lambda \frac{J_{1}\left(\sqrt{-\lambda\left(\xi-\xi_{1}\right)^{2}}\right)}{\sqrt{-\lambda\left(\xi-\xi_{1}\right)^{2}}} u\left(\xi_{1},-\xi_{1}\right)
\end{gathered}
$$

as $J_{0}(0)=1$.

From the last relation it follows that

$$
u(\xi,-\xi)=\left.u\right|_{A_{0} A}+\left.N[u]\right|_{A_{0} A}=u(\xi,-\xi)+\left.N[u]\right|_{\eta=-\xi},
$$

i.e.

$$
\begin{gathered}
N[u]=\int_{0}^{\xi} J_{0}\left(\left.\sqrt{\left.4 \lambda\left(\xi-\xi_{1}\right)^{2}\right)} \frac{\partial u}{\partial \eta_{1}} u\left(\xi,-\eta_{1}\right)\right|_{\eta_{1}=\xi} d \xi_{1}+\right. \\
+2 \lambda \int_{0}^{\xi} \frac{J_{1}\left(\sqrt{-4 \lambda\left(\xi-\xi_{1}\right)^{2}}\right)}{\sqrt{-4 \lambda\left(\xi-\xi_{1}\right)^{2}}} u\left(\xi_{1},-\xi_{1}\right) d \xi_{1}=0 .
\end{gathered}
$$

The boundary condition (29) is the lateral boundary condition of the telegraph potential on $A_{0} A: \eta=-\xi$, $0<\xi<1 / 2$.

If $\lambda=0$, then from (29) by differentiating by parts, we obtain the lateral boundary conditions for the one-dimensional Cauchy wave potential in the case of T.Sh. Kalmenov, D. Suragan [3].

Similarly, we find the boundary conditions on $B_{0} B$ :

$$
\begin{aligned}
& \left.N[u]\right|_{B_{0} B}=-\int_{\frac{1}{2}}^{\xi} J_{0}\left(\sqrt{4 \lambda\left(\xi-\xi_{1}\right)^{2}}\right) \frac{\partial u}{\partial \xi}\left(\xi_{1}, 1-\xi_{1}\right) d \xi_{1}+ \\
& +2 \lambda \int_{\frac{1}{2}}^{\xi} \frac{J_{1}\left(\sqrt{-4 \lambda\left(\xi-\xi_{1}\right)^{2}}\right)}{\sqrt{-4 \lambda\left(\xi-\xi_{1}\right)^{2}}}\left(\xi-\xi_{1}\right) u\left(\xi_{1}, 1-\xi_{1}\right) d \xi_{1}=0 .
\end{aligned}
$$

Thus, the lateral boundary conditions $N[u]$ on $A_{0} A$ and $B_{0} B$ are given by formulas (29), (30), respectively. 
Conversely. Let $\vartheta \in C^{2}(\bar{\Omega})$ satisfy equation (4), homogeneous initial conditions (5)-(6) and the lateral boundary conditions $(29)-(30)$. Let $u(\xi, \eta)$ be the telegraph potential defined by (9), then $\omega=u-\vartheta$ satisfies the homogeneous equation (4) and the homogeneous initial conditions (5)-(6).

By virtue of (24) and (31), we have

$$
\begin{aligned}
& \omega+\left.N[\omega]\right|_{A_{0} A}=\left.\omega\right|_{A_{0} A}=0, \\
& \omega+\left.N[\omega]\right|_{B_{0} B}=\left.\omega\right|_{B_{0} B}=0 .
\end{aligned}
$$

From the uniqueness of the solution of the mixed Cauchy problem we have $\omega=u-\vartheta \equiv 0$, i.e. $u=\vartheta$. By continuation of the solution outside the square under consideration, we see that $\left.N[u]\right|_{x=0}=0$, and $\left.N[u]\right|_{x=1}=0$.

Theorem 1 is completely proved.

\section{Telegraph potential solitons}

In a quarter of the plane $\Omega=\{x \geq 0, t \geq 0\}$ we consider the Cauchy problem for a homogeneous telegraph equation

$$
\begin{gathered}
L u(x, t)=\square u(x, t)-\lambda u(x, t)=\left(\frac{\partial^{2}}{\partial t^{2}}-\frac{\partial^{2}}{\partial x^{2}}\right) u(x, t)-\lambda u(x, t)=0 ; \\
\left.u(x, t)\right|_{t=0}=\tau(x), \\
\left.\frac{\partial u(x, t)}{\partial t}\right|_{t=0}=\nu(x) .
\end{gathered}
$$

We assume that $\tau(x) \equiv \nu(x) \equiv 0$ at $x \leq 0$ and we seek a solution in the whole half-space $t \geq 0$. It is natural, that it is determined by the Cauchy data $\tau(x)$ and $\nu(x)$ at $x \geq 0$.

By $\widetilde{\tau}(x)$ and $\widetilde{\nu}(x)$ we denote the functions

$$
\begin{aligned}
& \widetilde{\tau}(x)= \begin{cases}\tau(x), & x \geq 0, \\
0, & x \leq 0,\end{cases} \\
& \widetilde{\nu}(x)= \begin{cases}\nu(x), & x \geq 0, \\
0, & x \leq 0 .\end{cases}
\end{aligned}
$$

The solution of the Cauchy problem $u(x, t)$ is determined by the Riemann formula (see [22; 174])

$$
\begin{gathered}
u(x, t)=\frac{1}{2} u\left(x_{0}, 0\right) R\left(x_{0}, 0, x, t\right)+\frac{1}{2} u\left(x_{1}, 0\right) R\left(x_{1}, 0, x, t\right)+ \\
+\frac{1}{2} \int_{x_{0}}^{x_{1}}\left(\frac{\partial}{\partial \eta_{1}} u\left(\xi_{1}, 0\right) R\left(\xi_{1}, 0, x, t\right)-u\left(\xi_{1}, 0\right) \frac{\partial}{\partial \eta_{1}} R\left(\xi_{1}, 0, x, t\right)\right) d \xi_{1}= \\
=\frac{1}{2} \tau\left(x_{0}\right) R\left(x_{0}, 0, x, t\right)+\frac{1}{2} \tau\left(x_{1}\right) R\left(x_{1}, 0, x, t\right)+ \\
+\frac{1}{2} \int_{x_{0}}^{x_{1}}\left(\nu\left(\xi_{1}\right) R\left(\xi_{1}, 0, x, t\right)-\tau\left(\xi_{1}\right) \frac{\partial}{\partial \eta_{1}} R\left(\xi_{1}, 0, x, t\right)\right) d \xi_{1}
\end{gathered}
$$

where $x_{0}=x-t, \quad x_{1}=x+t$.

At $x_{1}=0, x_{0}=-t<0$, taking into account that $\tau\left(x_{0}\right)=0$ and $\nu(x)=0$, from (37) it follows that

$$
\begin{gathered}
\tau(t)=u(0, t)=\left.\frac{1}{2} \tau(x+t) R(x+t, 0, x, t)\right|_{x=0}+ \\
+\left.\frac{1}{2} \int_{0}^{x_{1}}\left(\nu\left(\xi_{1}\right) R\left(\xi_{1}, 0, x, t\right)-\tau\left(\xi_{1}\right) \frac{\partial}{\partial \eta_{1}} R\left(\xi_{1}, 0, x, t\right)\right) d \xi_{1}\right|_{x=0}= \\
=\frac{1}{2} \tau(t) R(t, 0,0, t)+\frac{1}{2} \int_{0}^{t}\left(\nu\left(\xi_{1}\right) R\left(\xi_{1}, 0,0, t\right)-\tau\left(\xi_{1}\right) \frac{\partial}{\partial \eta_{1}} R\left(\xi_{1}, 0,0, t\right)\right) d \xi_{1} .
\end{gathered}
$$

Equality (38) is the lateral boundary condition for the surface wave potential. 
It follows

Lemma. Suppose that the Cauchy data $\tau(x), \quad \nu(x) \in C^{2}(-\infty, \infty)$ and $\tau(x)=u(x, 0) \equiv 0$, $\nu(x)=\frac{\partial u(x, 0)}{\partial t} \equiv 0$ at $x \leq 0$. Then the surface telegraph potential $u(x, t)$ for $x=0$ satisfies the lateral boundary condition (38).

It is easy to verify that for $\lambda=0$ the condition (37) becomes a boundary condition of the surface wave potential.

Theorem 2. Suppose that the hypothesis of Lemma holds and $\lambda=0$ for $x \geq d>0$. Then the surface telegraph potential $u(x, t)$ at $x \rightarrow \infty$ turns into a soliton solution, i.e. $\lim _{x \rightarrow \infty} u(x, t)=\varphi(x-t)$ if and only if the condition is fulfilled

$$
\left.\left(\frac{\partial}{\partial t}-\frac{\partial}{\partial x}\right) u(x, t)\right|_{x=d}=0
$$

Proof. It is not difficult to show that if $\lambda=0$ at $x \geq d>0$, then the solution of the homogeneous telegraph equation given by $(37)$ can be represented in the form

$$
u(x, t) \equiv \psi(x+t)+\varphi(x-t) .
$$

Then

$$
\left.\left(\frac{\partial}{\partial t}-\frac{\partial}{\partial x}\right) u(x, t)\right|_{x=d}=\left.\psi^{\prime}(d+t)\right|_{x=d}=0 .
$$

Taking this into account, from (40) it follows that $\lim _{x \rightarrow \infty} u(x, t)=\varphi(x-t)$.

Theorem 2 is proved.

This paper was published under projects AP05133239, AP05134615, BR05236656 of the Science Committee of the Ministry of Education and Science of the Republic of Kazakhstan.

\section{References}

1 Kal'menov, T.Sh., \& Suragan, D. (2009). On spectral problems for the volume potential. Doklady Mathematics. 80:2, 646-649. DOI: 10.1134/S1064562409050032.

2 Kal'menov, T.Sh., \& Tokmagambetov, N.E. (2013). On a nonlocal boundary value problem for the multidimensional heat equation in a noncylindrical domain. Siberian Mathematical Journal, 54:6, 10231028. DOI: $10.1134 /$ S0037446613060086.

3 Kal'menov, T.Sh., \& Suragan, D. (2014). Initial-boundary value problems for the wave equation. Electronic Journal of Differential Equations, 2014:48, 1-6.

4 Kal'menov, T.Sh., \& Arepova, G.D. (2015). On a boundary condition of the surface heat potential. AIP Conf. Proc. 1676, 020054, 1-4.

5 Kal'menov, T.Sh., \& Arepova, G.D. (2016). On a heat and mass transfer model for the locally inhomogeneous initial data. Bulletin SUSU MMCS, 9:2, 124-129.

6 Kal'menov, T.Sh., Otelbaev, M., \& Arepova, G.D. (2018). BitsadzeSamarskii Boundary Condition for Elliptic-Parabolic Volume Potential. Doklady Mahematics, 480:2, 141-144.

7 Kal'menov, T.Sh., \& Suragan, D. (2011). A boundary condition and Spectral Problems for the Newton Potentials. Operator Theory: Advances and Applications, 216, 187-210.

8 Sadybekov, M., \& Oralsyn, G. (2018). On trace formulae of the generalised heat potential operator. Journal of Pseudo-Differential Operators and Applications, 9:1, 143-150.

9 Kal'menov, T.Sh., Arepova, G.D., \& Suragan, D. (2017). On the Symmetry of the Boundary Conditions of the Volume Potential. AIP Conference Proceedings, 1880, 040014.

10 Kal'menov, T.Sh., \& Arepova, G.D. (2016). On a heat and mass transfer model for the locally inhomogeneous initial data. AIP Conference Proceedings, 1789, 040028.

11 Kal'menov, T.Sh., \& Sadybekov, M. (2017). On a Frankl-type problem for a mixed parabolic-hyperbolic equation. Siberian Mathematical Journal, 58:2, 227-231.

12 Kal'menov, T.Sh., \& Sadybekov, M. (2018). Spectral properties of a Frankle type problem for parabolichyperbolic equations. EJDE. 
13 Arepova, G.D. (2016). On a regular problem for an elliptic-parabolic equation with a potential boundary condition. AIP Conference Proceedings, 1759, 020041.

14 Kal'menov, T.Sh., \& Arepova, G.D. (2016). On a heat transfer model for a locally inhomogeneous initial data. AIP Conference Proceedings, 1759, 020026.

15 Soldatov, A.P. (2014). Generelized potentials of double layer in plane theory of elasticity. Eurasian Math. Journal, 5:2, 78-125.

16 Kal'menov, T.Sh., \& Otelbaev, M. (2016). Boundary criterion for integral operators. Doklady Mahematics, 1(93), 58-61.

17 Kal'menov, T.Sh., \& Iskakova, U.A. (2007). A criterion for the strong solvability of the mixed cauchy problem for the Laplace equation. Doklady Mathematics, 75:3, 370-373.

18 Kal'menov, T.Sh., \& Iskakova, U.A. (2008). A method for solving the Cauchy problem for the Laplace equation. Doklady Mathematics, 78:3, 874-876.

19 Kal'menov, T.Sh., Sadybekov, M.A., \& Iskakova U.A. (2016). On a criterion for the solvability of one ill-posed problem for the biharmonic equation. Journal of Inverse and Ill-Posed Problems, 24:6, 777-783.

20 Koshlyakov, N.S., Gliner, E.B., \& Smirnov, M.M. (1970). Partial differential equations of mathematical physics. Moscow.

21 Vladimirov, V.S. (1981). Equations of Mathematical Phisycs. Moscow.

22 Bitsadze, A.V. (1982). Equations of Mathematical Phisycs. Moscow.

Т.Ш. Кәлменов, Г.Д. Арепова

\section{Телеграф теңдеуінің солитон шешімдерінің бар болуының қажетті және жеткілікті шарты}

Мақалада телеграф теңдеуі қарастырылған. Төртбұрышты облыс жағдайында Коши потенциалының бүйір шекараларында шекаралық шарттары табылған. Теңдеуді бірінші квадрантта қарастырғанда солитон шешімдерінің бар болуының қажетті және жеткілікті шарты алынған.

Kiлm сөздер: телеграф теңдеуі, телеграф потенциалы, іргелі шешім, солитон шешімі, локалды емес шекаралық шарты, үйірткі.

Т.Ш. Кальменов, Г.Д. Арепова

\section{Критерий существования солитонных решений телеграфного уравнения}

В статье рассмотрено телеграфное уравнение. Для случая прямоугольной области найдены краевые условия потенциала Коши на боковых границах. При рассмотрении уравнения в первом квадранте получен критерий существования солитонных решений.

Ключевые слова: телеграфное уравнение, телеграфный потенциал, фундаментальное решение, солитонное решение, нелокальные граничные условия, свертка. 